\title{
REGULAR OVERRINGS OF REGULAR LOCAL RINGS
}

BY

\section{JUDITH SALLY}

\begin{abstract}
The local factorization theorem of Zariski and Abhyankar characterizes all 2-dimensional regular local rings which lie between a given 2-dimensional regular local $r$ ing $R$ and its quotient field as finite quadratic transforms of $R$. This paper shows that every regular local ring $R$ of dimension $n>2$ has inf initely many minimal regular local overrings which cannot be obtained by a monoidal transform of $R$. These overrings are localizations of rings generated over $R$ by certain quotients of elements of an $R$-sequence. Necessary and sufficient conditions are given for this type of extension of $R$ to be regular.
\end{abstract}

1. Introduction. The factorization theorem of Zariski and Abhyankar [1] characterizes all 2-dimensional regular local rings which lie between a given 2dimensional regular local ring $R$ and its quotient field as finite quadratic transforms of $R$. There are two key steps in the proof:

(I) the existence of a quadratic transform of $R$ in any such overring $T$ or, equivalently (for the purpose of our generalizing the statement to higher dimensions), the existence in $T$ of a quotient of two basis elements of the maximal ideal of $R$;

(II) the fact that the union of an infinite sequence of quadratic transforms of $R$ is a valuation domain.

Hironaka [3, p. 151] states that the corresponding global theorem is false for nonsingular varieties of dimension $n>2$. Theorem 4.4 below shows that every $n$ dimensional regular local ring $(n>2)$ has infinitely many regular local overrings failing (I). (It is clear that statement (II) is false for $n>2$.) However, there are other finiteness conditions which are satisfied in higher dimensions. In Theorem 5.1 we give examples of two distinct types of minimal regular local overrings of a given $n$-dimensional regular local ring $(n>2)$. Theorem 5.3 proves that if $R$ is an $n$-dimensional regular local ring with the property that any $n$-dimensional integrally closed locality over $R$, contained in the quotient field of $R$, is analytically irreducible, then every $n$-dimensional integrally closed local overring is a locality over $R$. Some of the proofs of these theorems use the results of Theorem 3.1 which is of independent interest because it gives necessary and sufficient conditions for certain extensions of a regular local ring to be globally regular.

Received by the editors May 19, 1971.

AMS 1970 subject classifications. Primary 13H05, 14A05, 14B05.

Key words and phrases. Regular local ring, overring, valuation domain, monoidal and quadratic transforms of a regular local ring, dimension formula, $R$-sequence, locality, analytically irreducible domain. 
The results contained in this paper form part of my doctoral thesis. It is with much pleasure that I acknowledge my gratitude to Professor Irving Kaplansky.

2. Preliminaries. Let $R$ be a Noetherian domain and $\left(x, x_{1}, \cdots, x_{i}\right)$ an ideal of $R$, where $x \neq 0$ and $i \geq 1$. Let $t_{1}, \cdots, t_{i}$ be indeterminates and $\phi$ the homomorphism of $R\left[t_{1}, \cdots, t_{i}\right]$ onto $T=R\left[x_{1} / x, \cdots, x_{i} / x\right]$ defined by $\phi\left(t_{j}\right)=x_{j} / x$ for $j=1, \ldots, i$, and $\phi$ is the identity on $R$. It is well known that if $x, x_{1}, \ldots$, $x_{i}$ is an $R$-sequence then kernel $\phi=\left(x t_{1}-x_{1}, \ldots, x t_{i}-x_{i}\right)$. Davis [2] proves that if $\left(x, x_{1}, \cdots, x_{i}\right)$ is an ideal of rank $i+1$ then the dimension formula holds between $R$ and $T$, i.e., if $Q$ is a prime ideal of $T$ and $Q \cap R=P$ then rank $P=$ rank $Q+$ tr.d. $T / Q: R / P$.

With the same notation as above, if $P$ is a prime ideal of $R$ and $P^{*}$ its extension to $R\left[t_{1}, \cdots, t_{i}\right]$ then $\operatorname{ker} \phi \subset P$ implies that $P T$ is a prime ideal of $T$, $T / P T \cong R / P\left[t_{1}, \cdots, t_{i}\right]$ and tr.d. $T / P T: R / P=i$. If, in addition, we assume that $R$ is an $n$-dimensional regular local ring and $x, x_{1}, \ldots, x_{i}$ is an $R$-sequence then $T=R\left[x_{1} / x, \cdots, x_{i} / x\right]$ is $n$-dimensional. If $N$ is a maximal ideal of $T$ then $\operatorname{rank} N=n$ or $n-1$ and $\operatorname{rank} N=\operatorname{rank} N \cap R$.

Throughout the remainder of this paper $\phi$ denotes the map of $R\left[t_{1}, \cdots,{ }_{i}\right]$ onto $T$ defined above and $Q^{\prime}$ denotes the inverse image in $R\left[t_{1}, \ldots, t_{i}\right]$ of an ideal $Q$ of $T$. The basic terminology is that of Kaplansky [4] and Nagata [8]. We use the phrase " $T$ is an overring of a domain $R$ " to mean that $T$ contains $R$ and is contained in the quotient field of $R$. We use a restricted form of the definition of monoidal transform as follows.

Definition 2.1. Let $(R, M)$ be an $n$-dimensional regular local ring. Let $P$ be a nonzero prime ideal of $R$ such that $R / P$ is regular. The localization, at a prime ideal containing $M$, of the ring generated over $R$ by $P / x$, for some nonzero $x \in P$, is called a monoidal transform of $R$; if $P=M$ it is called a quadratic trans. form of $R$.

3. Regular extensions of a regular local ring. Let $(R, M)$ be an $n$-dimensional regular local ring. Let $x, x_{1}, \cdots, x_{i}$ be an $R$-sequence. Theorem 3.1 gives necessary and sufficient conditions for $T=R\left[x_{1} / x, \ldots, x_{i} / x\right]$ to be a regular domain. David Whitman [10] proved that if $\left\{x, x_{1}, \cdots, x_{i}\right\}$ is a subset of a regular system of parameters of $R$, i.e., a subset of a minimal basis for $M$, then $T$ is regular. (Also see [5, p. 390].) We will show that there is one and only one other type of $R$-sequence such that the resulting $T$ is regular.

Theorem 3.1. Let $(R, M)$ be an $n$-dimensional regular local ring, $n>1$. Let $x, x_{1}, \cdots, x_{i}$ be an $R$-sequence and $T=R\left[x_{1} / x, \cdots, x_{i} / x\right]$. Then $T$ is an $n$ dimensional regular domain if and only if one of the following holds:

(a) the elements $x, x_{1}, \ldots, x_{i}$ form a subset of a minimal basis for $M$,

(b) (1) $x \in M^{2}$ and $x_{j} \notin M^{2}$ for $i \leq j \leq i$, and 
(2) if $P$ is the contraction in $R$ of a rank $n-1$ maximal ideal of $T$ containing $x$, then either the elements $x, x_{1}, \cdots, x_{i}$ form a subset of a minimal bas is for $P_{P}$ in $R_{P}$ or $x \in P^{(2)}$.

Proof. Assume that $T=R[t] /(\operatorname{ker} \phi)$ is a regular domain. If for some $j, 1 \leq$ $j \leq i$, the element $x_{j}$ is in $M^{2}$, then $x t_{j}-x_{j}$ is in the square of the maximal ideal $(M, t) R[t]$. This contradicts the fact that $R[t]_{(M, t)} /(\operatorname{ker} \phi)_{(M, t)}$ is regular, so we have that the elements $x_{1}, \cdots, x_{i}$ are not in $M^{2}$. If (a) does not hold, there is a relation $a_{0} x+a_{1} x_{1}+\cdots+a_{i} x_{i} \in M^{2}$, where the $a_{i}$ are in $R$ but not all in $M$. Suppose that there is a $j, 1 \leq j \leq i$, such that $\alpha_{j} \notin M$. Then

$$
\begin{aligned}
a_{1}\left(x t_{1}-x_{1}\right)+ & \cdots+a_{j}\left(x t_{j}-x_{j}\right)+\cdots+a_{i}\left(x t_{i}-x_{i}\right) \\
& =a_{1} x t_{1}+a_{2} x t_{2}+\cdots+a_{j} x\left(t_{j}+a_{0} / \alpha_{j}\right)+\cdots+a_{i} x t_{i}+y,
\end{aligned}
$$

where $y \in M^{2}$. But this relation shows that the generators of $\operatorname{ker} \phi$ are linearly dependent modulo $N^{2}$, where $N$ is the maximal ideal

$$
\left(M, t_{1}, \cdots, t_{j}+a_{0} / a_{j}, \cdots, t_{i}\right) R[t] .
$$

Hence all the elements $\alpha_{1}, \ldots, \alpha_{i}$ must be in $M$, and $x$ is in $M^{2}$. Suppose, in addition, that $P$ is the contraction of a rank $n-1$ maximal ideal of $T$ containing $x$. The fact that $T_{R-P}=R_{P}\left[x_{1} / x, \ldots, x_{i} / x\right]$ is regular implies, by the above, that either the elements $x, x_{1}, \cdots, x_{i}$ form a subset of a minimal basis for $P_{P}$ in $R_{P}$ or $x \in P^{(2)}$.

Conversely, to prove that (a) implies that $T$ is regular we cite the result of Whitman [10]. Assume instead that (b) holds. First we show, by induction on $i$, that (b)(1) implies that $T_{N}$ is regular for all rank $n$ maximal ideals $N$ of $T$. Let $i=1$. Suppose, on the contrary, that $N$ is a rank $n$ maximal ideal such that $T_{N}$ is not regular. Now, $\left(N^{\prime}\right)^{2} \cap R=M^{2}$. Hence $x t_{1}-x_{1} \in\left(N^{\prime}\right)^{2}$ and $x \in M^{2}$ imply that $x_{1} \in M^{2}$, a contradiction. Assume that $i>1$, and let $S=R\left[x_{1} / x, \cdots, x_{i-1} / x\right]$. If $N$ is a rank $n$ maximal ideal of $T$ then $N \cap S=Q$ has rank $n$, so the induction hypothesis implies that $S_{Q}$ is regular. Now,

$$
T_{N}=S_{Q}\left[t_{i}\right]_{N_{(S-Q)}^{\prime \prime}} /\left(x t_{i}-x_{i}\right) N_{(S-Q)}^{\prime \prime}=S\left[t_{i}\right]_{N^{\prime \prime}} /\left(x t_{i}-x_{i}\right)_{N}^{\prime \prime},
$$

where $N^{\prime \prime}$ is the inverse image of $N$ in $S\left[t_{i}\right]$. If $T_{N}$ is not regular and $x t_{i}-x_{i}$ is in $\left(N^{\prime \prime}\right)^{2}$, then $x_{i}$ is in $Q^{2}$. But $\phi: R\left[t_{1}, \cdots, t_{i-1}\right] \rightarrow S$ is the identity on $R$, so that $\left(Q^{2}\right)^{\prime} \cap R=\left(Q^{\prime}\right)^{2} \cap R=M^{2}$ and $x_{i} \in M^{2}$, the desired contradiction. Thus we have shown that (b)(1) implies that $T_{N}$ is regular for all rank $n$ maximal ideals $N$. To complete the proof that $T$ is regular, since $T$ is obviously Noetherian, we just need to check that $T_{N}$ is regular for any rank $n-1$ maximal ideal $N$. This follows immediately if $x \notin N$, because, in that case, $T_{N}=R_{N \cap R}$. Assume that $x \in N$. Then $P=N \cap R$ has rank $n-1$ and $N_{R-P}$ is a rank $n-1$ maximal ideal 
of $R_{P}\left[x_{1} / x, \cdots, x_{i} / x\right]$. Now, by hypothesis, either the elements $x, x_{1}, \cdots, x_{i}$ form a subset of a minimal basis for $P_{P}$ in $R_{P}$, in which case $T_{N}$ is regular by [10], or $x \in P^{(2)}$. If $x \in P^{(2)}$, then $\left(R_{P}, P_{P}\right)$ satisfies $(b)(1)$ so that $T_{N}$ is regular. This completes the proof of the theorem.

Example 3.2. Let $(R, M)$ be an $n$-dimensional regular local ring, and $x$ and $y$ be elements which form a subset of a minimal basis for $M$ in $R$. Then $R\left[y^{2} / x\right]$ is integrally closed but not regular.

Example 3.3. With the notation as in Theorem 3.1, let $M=\left(x, x_{1}, \cdots, x_{n-1}\right)$ and $T=R\left[x_{1} / x, \ldots, x_{i} / x\right]$. Assume $i \leq n-1$. Then for every $k \geq 2$, the ideals

$$
M_{k}=\left(x-x_{1}^{k}, x_{2}, \cdots, x_{n-1},\left(x_{1}^{k} / x\right)-1, x_{2} / x, \ldots, x_{i} / x\right)
$$

are distinct rank $n-1$ maximal ideals of $T$. Assume $i<n-1$, then for every $k>0$, the ideals

$$
N_{k}=\left(x, x_{1}, \ldots, x_{n-2}, x_{n-1}^{k}\left(x_{1} / x\right)-1, x_{2} / x, \ldots, x_{i} / x\right)
$$

are distinct rank $n-1$ maximal ideals of $T$ containing $x$.

Example 3.4. Using the notation of Theorem 3.1, we observe that if $T=T_{i}$ is (a)-regular, then all the rings $T_{j}=R\left[x_{1} / x, \ldots, x_{j} / x\right]$ for $j<i$ are regular. The following example shows that if $T$ is (b)-regular then $T_{j}$ for $j<i$ need not be regular. $T=T_{i}$ being (b)-regular implies that $T_{i-1}$ localized at a rank $n$ maximal ideal is regular but it does not imply regularity at all rank $n-1$ maximal ideals. The reason for this failure is that, while the rank $n$ maximal ideals of $T_{i-1}$ have (infinitely many) maximal ideals of $T$ lying over them, a rank $n-1$ maximal ideal of $T_{i-1}$ containing $x$ but not $x_{i}$ has no prime ideal of $T$ lying over it. Let $R=k[u, v, w]_{(u, v, w)}$, where $k$ is a field and $u, v, w$ are indeterminates. Let $T=R\left[u /\left(u v-w^{2}\right), v /\left(u v-w^{2}\right)\right]$. Then $T$ is regular but $R\left[u /\left(u v-w^{2}\right)\right]$ is not.

Example 3.5. The (a)-regular rings of Theorem 3.1 are unique factorization domains as is easily seen by applying Nagata's theorem [7]. A (b)-regular ring is not necessarily a UFD. Let $(R, M)$ be a 2-dimensional regular local ring; say $M=(x, y)$. Let $f$ be a principal prime of the form $f=a x+b y$, where $0 \neq a \in M$. Then $T=R[y / f]$ is not a UFD. If $b \in M, T$ is regular, otherwise not.

The following corollary is a global version of Theorem 3.1 and the proof follows directly from that theorem by localization.

Corollary 3.6. Let $R$ be an n-dimensional regular domain, $n>1$. Let $x, x_{1}$, $\cdots, x_{i}$ be an $R$-sequence. Then $T=R\left[x_{1} / x, \ldots, x_{i} / x\right]$ is regular if and only if for all maximal ideals $N$ of $T$ containing $x$, if $P=N \cap R$, then either $x_{1} x_{1}, \ldots$, $x_{i}$ form a subset of a minimal basis for $P_{P}$ in $R_{P}$ or $x \in P^{(2)}$ and $x_{1}, \ldots, x_{i}$ $\notin P^{(2)}$.

As an application of Corollary 3.6 we will see that many examples of globally 
regular rings can be obtained as extensions of a given regular local ring using quotients of elements taken from a set of generators of the maximal ideal. To describe these extensions neatly we use a little graph theory. Let $R$ be an $n$-dimensional regular local ring with maximal ideal $M=\left(x_{1}, \ldots, x_{n}\right)$. Let $T=$ $R\left[y_{1} / y_{2}, y_{3} / y_{4}, \cdots, y_{2 k-1} / y_{2 k}\right]$ where for $1 \leq i \leq 2 k, y_{i}=x_{j}$ for some $j$. We assume $y_{i} / y_{i+1} \neq 1$. Let $G$ be the graph defined as follows. The distinct $y_{i}$ are the points of $G$, each $y_{i} / y_{i+1}$ represents an oriented edge, $y_{i} \rightarrow y_{i+1}$, of $G$ and, if $y_{i} / y_{k}$ is a product of the generators of $T$ over $R$, the edge $y_{i} \rightarrow y_{k}$ is also in $G$. We say that $T$ is a $G$-extension of $R$ if (1) $G$ contains no point with more than one edge emanating from it, and (2) $G$ contains no cycles. For example, if $R$ is a 4-dimensional regular local ring with maximal ideal $M=(x, y, z, w)$, then $R[x / y, z / w]$ and $R[x / y, z / y, y / w]$ are $G$-extensions of $R$. Proposition 3.8 states that $G$-extensions of $R$ are regular UFD's.

It is easy to check that if $T$ is a $G$-extension of $R$ then the set $f_{i}=y_{i} / y_{i+1}$ of fractions which generate $T$ over $R$ can be "nicely ordered", that is, ordered in such a way, say $f_{i_{1}}, \cdots, f_{i_{k}}$, that the numerator of $f_{i_{b}}$, for $1 \leq b \leq k$, is not the denominator of $f_{i_{b+j}}$ for $j \geq 0$. Using this fact, although several technical details have to be dealt with (Lemma 3.7), the main technique of the proof of Proposition 3.8 is that of the previous theorem. For this reason we omit it.

Lemma 3.7. Let $T=R\left[y_{1} / y_{2}, y_{3} / y_{4}, \cdots, y_{2 k-1} / y_{2 k}\right]$ be a G-extension of $R$. Let $P=\left(x_{j_{1}}, \ldots, x_{j_{q}}\right)$ be a prime ideal generated by a subset of the generators $x_{1}, \cdots, x_{n}$ of $M$. If, for $1 \leq b \leq q, x_{j_{b}} \neq y_{2 i-1}$, where $1 \leq i \leq k$, then $P T$ is $a$ prime ideal of $T$ generated by an $R$-sequence.

Proposition 3.8. Let $T$ be a G-extension of $R$. Then $T$ is an $n$-dimensional regular UFD.

4. Simple extensions and accessible domains.

Definition 4.1. Let $(R, M)$ be an $n$-dimensional regular local ring, $n>1$. T is a simple extension of $R$ if $T$ is the localization at a rank $n$ prime of a ring generated over $R$ by a quotient of two elements of $R$ which form a subset of a minimal basis for $M$ in $R$. An $n$-dimensional regular local overring $T$ of $R$ is acces. sible from $R$ if $T$ can be obtained from $R$ by a finite sequence of simple extensions.

In this terminology, the factorization theorem of Zariski and Abhyankar [1] states that if $R$ is a 2-dimensional regular local ring then every 2-dimensional regular local overring of $R$ is accessible from $R$. For dimension $n>2$, the following theorem shows that every $n$-dimensional regular local ring $R$ has infinitely many regular local overrings which are not accessible from $R$. First we need two lemmas giving information about the structure of regular local extensions of the type $R[a / b]_{N}$ with $a \notin M^{2}$ and $b \in M^{2}$. 
Lemma 4.2. Let $(R, M)$ be an n-dimensional regular local ring, $n>1$. Let $T=R[a / b]$, where $a$ and $b$ are nonzero, relatively prime elements of $R$ with $a \notin M^{2}$ and $b \in M^{2}$. If $x_{2}, \cdots, x_{n}$ are any elements of $R$ which, with $a$, form $a$ minimal basis for $M$, then $x_{2}, \cdots, x_{n}$ form a subset of a minimal basis for $N_{N}$ in $T_{N}$, where $N$ is any rank $n$ maximal ideal of $T$.

Proof. Write $b=a_{1} a+a_{2} x_{2}+\cdots+a_{n} x_{n}$ with all $\alpha_{i}$ in $M$. Given a rank $n$ maximal ideal $N$ of $T$, let $s=1-a_{1}(a / b)$. Then $s \in T-N$ and $s a \epsilon$ $\left(x_{2}, \cdots, x_{n}\right) T$. Consequently, $N_{N}=\left(x_{2}, \cdots, x_{n}, f(a / b)\right) T_{N}$.

Lemma 4.3. Let $(R, M)$ be an n-dimensional regular local ring, $n>1$, and let $a$ and $b$ be nonzero, relatively prime elements of $R$. Let $T=R[a / b]_{N}$, where $a \notin M^{2}$ and $N$ is any rank $n$ maximal ideal of $R[a / b]$. If $x_{1}, \cdots, x_{n}$ is any minimal basis for $M$ then, for $i \neq j$, the quotient $x_{i} / x_{j}$ is in $T$ if and only if $(a, b) R$ $\subseteq\left(x_{i}, x_{j}\right) R$.

Proof. Since $a \in M-M^{2}$, we can assume that $a=x_{1}+\beta_{2} x_{2}+\cdots+\beta_{n}{ }^{x}$, with $\beta_{i} \in R$. Suppose, for $i \neq j$, that $x_{i} / x_{j}$ is in $T$. Then $i$ or $j$ must be 1 because, by Lemma $4.2, x_{2}, \cdots, x_{n}$ form an $R$-sequence in any order in $T$. We may assume that $x_{1} / x_{j}$ is in $T$ because $x_{j} / x_{1} \in T$ implies that $x_{1} / x_{j} \in T$ since $x_{j} T$ is a prime ideal. Now $x_{1} \in x_{j} T$ implies that $\left(x_{1}, x_{j}\right) R$ is contained in $x_{j} T \cap R$. (Actually, $\left(x_{1}, x_{j}\right) R=x_{j} T \cap R$ because the dimension formula holds between $R$ and T.) Let $J$ be the inverse image of $x_{j} T$ in $R[t]$, where $t$ is an indeterminate. Then rank $J=\operatorname{rank} x_{j} T+1$. Now $\left(x_{1}, x_{j}\right) R \subseteq x_{j} T$ implies that $\left(x_{1}, x_{j}\right)^{*}$, the rank 2 extension of $\left(x_{1}, x_{j}\right) R$ to $R[t]$, is contained in $J$. Thus we have $\left(x_{1}, x_{j}\right)^{*}=J$ and $(b t-a) R[t]$ is contained in $\left(x_{1}, x_{j}\right)^{*}$, proving that $(a, b) R \subseteq\left(x_{1}, x_{j}\right) R$.

Conversely, if $(a, b) R \subseteq\left(x_{i}, x_{j}\right) R$, then $a=x_{1}+\beta_{2} x_{2}+\cdots+\beta_{n} x_{n}$ implies that $i$ or $j=1$. Now $(a, b) R \subseteq\left(x_{1}, x_{j}\right) R$ means that $(a t-b) R[t] \subset\left(x_{1}, x_{j}\right)^{*}$, so that $\left(x_{1}, x_{j}\right) T$ is a rank 1 prime of $T$. Therefore $\left(x_{1}, x_{j}\right) T=x_{j} T$ because $x_{j} T$ is a principal prime of $T$. Hence $x_{1} / x_{j} \in T$.

Theorem 4.4. Let $(R, M)$ be an $n$-dimensional regular local ring, $n>1$. Let $a$ and $b$ be nonzero, relatively prime elements of $R$ with $a \notin M^{2}$ and $b \in M^{2}$. Let $T=R[a / b]_{N}$, where $N$ is any rank $n$ maximal ideal of $R[a / b]$. If the ideal $(a, b) R$ is prime then no simple extension of $R$ is contained in $T$.

Note. The statement "the ideal $(a, b) R$ is prime" is equivalent to " $b$ is a prime of T."

Proof. This follows directly from Lemma 4.3. More specifically, suppose that $(a, b) R \subseteq(v, w) R$, where $v, w$ form a subset of a minimal basis for $M$. Then $(a, b) R=(v, w) R$. Clearly, we may assume $v=a$. Passing to the regular local ring $\bar{R}=(R / a R)_{M / a R}$ with maximal ideal $\bar{M}$, we have that $\bar{b}=\bar{w} \bar{R}$. But $\bar{b} \in \bar{M}^{2}$ 
and $\bar{w}$, being a generator of $\bar{M}$, cannot be in $\bar{M}^{2}$. This is a contradiction and completes the proof.

Corollary 4.5. Let $(R, M)$ be an n-dimensional regular local ring, $n>2$. Then $R$ bas infinitely many regular local overrings which fail to contain any simple extension of $R$.

Proof. Let $x, y, z$ be a subset of a minimal basis for $M$. Then, for example, since the elements $f_{i}=y^{2}+z^{2 i+1}$, where $i \geq 1$, are prime elements of $R$, the rings $T_{i}=R\left[x / f_{i}\right]_{N}$, where $N$ is any rank $n$ maximal ideal of $R\left[x / f_{i}\right]$, satisfy the hypotheses of the theorem.

We note that David Shannon has results in this same direction, which he announced in Notices Amer. Math. Soc. 18 (1971), p. 358, Abstract 683-A14.

Example 4.6. An accessible extension of $R$ can have a regular local subring which is not accessible from $R$. Let $(R, M)$ be an $n$-dimensional regular local ring, $n>2$. Let $M=\left(x, y, z, w_{4}, \cdots, w_{n}\right)$. Let

$$
S=R\left[x / y, z / y,\left(y+\left(z^{3} / y\right)\right) /(x / y)\right]_{Q},
$$

where $Q=\left(M, x / y, z / y,\left(\left(y+\left(z^{3} / y\right)\right) /(x / y)\right)-1\right)$. Let $T=R\left[x /\left(y^{2}+z^{3}\right)\right]_{N}$, where $N=Q \cap R\left[x /\left(y^{2}+z^{3}\right)\right]$. Then $R \subset T \subset S, S$ is accessible from $R$ and from $T$, but $T$ is not accessible from $R$.

It is interesting to note that the rings which provided examples of inaccessible overrings for $n>2$ have a very simple form for $n=2$.

Proposition 4.7. Let $(R, M)$ be a 2-dimensional regular local ring. Let $a$ and $b$ be nonzero, relatively prime elements of $R$ with $a \notin M^{2}$ and $b \in M^{2}$. Let $T=$ $R[a / b]_{Q}$, where $Q$ is any rank 2 maximal ideal. Then $T=R\left[a / x^{\nu}\right]_{N}$, where $x$ is any element of $R$ such that $M=(a, x)$, and $v$ is the value of the image of $b$ in the $D V R R / a R$.

Proof. Let $x$ be any element of $R$ such that $M=(a, x) . R / a R$ is a DVR and $\bar{b}=\bar{u} \bar{x}^{v}$ so we may write $b=u x^{v}+\gamma a$, where $u \notin M$ and $\gamma \in M$. Let $s=1-$ $\gamma\left(a /\left(u x^{v}+\gamma a\right)\right)$. Then $s \in R[a / b]-Q$ and $s\left(a / x^{v}\right) \in R[a / b]$, so that $a / x^{v} \in T$. Now $a / b=a /\left(u x^{v}+\gamma a\right)=\left(a / x^{v}\right) /\left(u+\gamma\left(a / x^{v}\right)\right)$. Thus $R[a / b] \subseteq R\left[a / x^{v}\right] Q \cap R\left[a / x^{v}\right]$ $\subseteq T=R[a / b]_{Q}$, and we have that $R\left[a / x^{\nu}\right]_{N}=T$, where $N=Q \cap R\left[a / x^{\nu}\right]$.

5. Some finiteness conditions. The set of minimal regular local overrings of a 2-dimensional regular local ring $R$ is precisely the set of immediate quadratic transforms of $R$. If the dimension of the regular local ring $R$ is greater than two, Theorem 5.1 below shows that simple extensions and the extensions constructed in Theorem 4.4 are two distinct types of minimal regular local overrings of $R$.

Theorem 5.1. Let $(R, M)$ be an n-dimensional regular local ring, $n>1$. Let $R[y / x]_{N}=T$ be a simple extension of $R$. Then there are no regular local rings 
properly between $R$ and $T$. The extensions $R[a / b]_{N}$ of Theorem 4.4 are also minimal regular local extensions of $R$, if $n>2$.

Before proving the theorem we will state a local version of Theorem 3.1 for the case $i=1$, the proof of which is entirely similar to the proof of Theorem 3.1 .

Lemma 5.2. Let $(R, M)$ be an $n$-dimensional regular local ring, $n>1$. Let $x$ and $y$ be nonzero, relatively prime elements of $R$. Let $T=R[y / x]_{N}$, where $N$ is a rank $n$ maximal ideal of $R[y / x]$. Then $T$ is a regular local ring if and only if either

(1) $x \in M^{2}$ and $y \notin M^{2}$, or

(2) $x \notin M^{2}$, and one of the following bolds:

(a) $x$ and $y$ form a subset of a minimal basis for $M$,

(b) $y \notin M^{2}$ and $N \neq\left(M,(y / x)+u^{-1}\right)$, where $u \in R-M$ and $x+u y \in M^{2}$,

(c) $y \in M^{2}$ and $N \neq(M, y / x)$.

Proof of Theorem 5.1. Suppose $\left(S, M_{1}\right)$ is a regular local ring properly lying between $R$ and the simple extension $T=R[y / x]_{N}$. We will derive a contradiction to Zariski's Main Theorem [8, p. 137] by showing that $M S=M_{1}$. Clearly, $y / x$ is not in $S$ so, using the fact that $x$ is a principal prime in $T$, we have that $x$ and $y$ are relatively prime in $S$. Therefore $S[y / x]_{N_{1}}=T$, where $N_{1}=N_{N} \cap S[y / x]$. Now, using Lemma 5.2, we see that $x$ and $y$ form a subset of a minimal basis for $M_{1}$ by ruling out all other possibilities. Since $T$ is a simple extension of $R$, if $M=\left(x, y, x_{3}, \cdots, x_{n}\right)$, then $x, x_{3}, \cdots, x_{n}$ are linearly independent $\bmod N_{N}^{2}$ in $T$. Suppose in $S$ there is a relation

$$
a_{1} x+a_{2} y+a_{3} x_{3}+\cdots+a_{n} x_{n} \in\left(M_{1}\right)^{2},
$$

where $a_{i} \in S$, not all $a_{i} \in M_{1}$. One of the $a_{i}$ for $i>2$ is a unit of $S$ so we have

$$
\left(\alpha_{1}+\alpha_{2}(y / x)\right) x+\alpha_{3} x_{3}+\cdots+\alpha_{n} x_{n} \in N_{N}^{2},
$$

which is a contradiction. Hence $x, y, x_{3}, \cdots, x_{n}$ generate $M_{1}$ and $M S=M_{1}$.

Suppose instead that $T=R[a / b]_{N}$ is as in Theorem 4.4. Then the hypothesis on $(a, b) R$ implies that $b$ is a prime element of $T$, and hence that $a$ and $b$ are relatively prime elements in any regular local ring $S$ which is assumed to lie between $R$ and $T$. Thus we have $S[a / b]_{N_{N} \cap S[a / b]}=T$. Applying Lemma 5.2 we see that $a \notin\left(M_{1}\right)^{2}$, where $M_{1}$ is the maximal ideal of $S$. Let $a, x_{2}, \cdots, x_{n}$ be a minimal basis for $M$ in $R$. Then, if there is a relation

$$
a_{1} a+a_{2} x_{2}+\cdots+a_{n} x_{n} \in\left(M_{1}\right)^{2},
$$

with $a_{i} \in S$, but not all in $M_{1}$, it is clear that some $\alpha_{j}$, for $j \neq 1$, is a unit of $S$. But $a=b(a / b)$ is in $N_{N}^{2}$, since $b$ is, so we have that $a_{2} x_{2}+\cdots+a_{n} x_{n}$ is in $N_{N}^{2}$, which is a contradiction to Lemma 4.2. Thus $M S=M_{1}$ and again we have a 
contradiction to Zariski's Main Theorem. Therefore no such $S$ can exist and $T$ must be minimal.

For a large class of $n$-dimensional regular local rings all $n$-dimensional, integrally closed local overrings are localities. It is an open question whether this "large class" includes all $n$-dimensional regular local rings.

Theorem 5.3. Let $(R, M)$ be an n-dimensional regular local ring with the property that any $n$-dimensional, integrally closed locality over $R$, which is contained in the quotient field of $R$, is analytically irreducible. Then every $n$-dimensional, integrally closed local overring of $R$ is a locality over $R$.

Proof. Let $T$ be an $n$-dimensional, integrally closed local overring of $R$. Let $N=\left(y_{1}, \cdots, y_{t}\right)$ be the maximal ideal of $T$. Set $S=R\left[y_{1}, \ldots, y_{t}\right]$. By Rees' Theorem [9], $S^{\prime}$, the integral closure of $S$, is a finitely generated $S$-module. Therefore, we have

$$
R \subseteq S \subseteq S^{\prime}=R\left[y_{1}, \cdots, y_{t}, w_{1}, \cdots, w_{k}\right] \subseteq T
$$

the last inclusion holds because $T$ is integrally closed. By assumption, $S_{N \cap S^{\prime}}^{\prime}$ is analytically irreducible, so by applying Zariski's Main Theorem, we have that $S_{N \cap S^{\prime}}^{\prime}=T$.

6. A remark on the relationship between Mac Lane's inductive values and quadratic transforms. Zariski and Abhyankar have also characterized every valuation overring of a given 2-dimensional regular local ring $R$ in terms of its quadratic transforms: either as a 1 -dimensional localization of a finite quadratic transform or as the union of an infinite sequence of quadratic transforms. For the special case $R=V[x]_{(\pi, x)}$, where $V$ is a discrete valuation ring with maximal ideal $\pi V$ and $x$ is an indeterminate, an earlier construction of S. Mac Lane [6] represents any rank 1 valuation $\nu$ of $R$ either as an "inductive value" or as a "limit value" of a sequence of approximations to $\nu$. The relationship between these characterizations seems to have gone unnoticed except for a remark of $M$. van der Put in his review (Zentralblatt, Band 198, Heft 2, Feb. 4, 1971, p. 366) of Inoue's paper extending Mac Lane's results. In fact, Mac Lane's inductive values are the $M$-adic valuations of suitable quadratic transforms of $R$ and his limit values are the valuations attached to unions of infinite sequences of quadratic transforms of $R$. The key polynomial which Mac Lane uses to define an approximation to $\nu$ is a multiple of the monic polynomial which is a generator of the center of the transform.

\section{BIBLIOGRAPHY}

1. S. Abhyankar, On the valuations centered in a local domain, Amer. J. Math. 78 (1956), 321-348. MR 18, 556.

2. E. Davis, Ideals of the principal class, $R$-sequences and a certain monoidal transformation, Pacific J. Math. 20 (1967), 197-205. MR $34 \# 5860$. 
3. H. Hironaka, Resolution of singularities of an algebraic variety over a field of characteristic zero. I, Ann. of Math (2) 79 (1964), 109-203. MR 33 \#7333.

4. I. Kaplansky, Commutative rings, Allyn and Bacon, Boston, Mass., 1970. MR 40 $\# 7234$.

5. S. Lichtenbaum, Curves over discrete valuation rings, Amer. J. Math 90 (1968), 380-405. MR $37 \# 6284$.

6. S. Mac Lane, $A$ construction for absolute values in polynomial rings, Trans. Amer. Math. Soc. 40 (1936), 363-395.

7. M. Nagata, A remark on the unique factorization theorem, J. Math. Soc. Japan 9 (1957), 143-145. MR 18, 869.

8. - Local rings, Interscience Tracts in Pure and Appl. Math., no. 13, Interscience, New York, 1962. MR 27 \#5790.

9. D. Rees, A note on analytically unramified local rings, J. London Math. Soc. 36 (1961), 24-28. MR 23 \#A3761.

10. D. G. Whitman, Some remarks on regular local rings, Math. Japon. 15 (1970), 1517. MR $43 \# 1971$.

DEPARTMENT OF MATHEMATICS, UNIVERSITY OF CHICAGO, CHICAGO, ILLINOIS 60637

Current address: Department of Mathematics, Northwestern University, Evanston, Illinois 60201 